\title{
When do semantic orienting tasks hinder recall?
}

\author{
EUGENE WINOGRAD \\ Emory University, Atlanta, Georgia 30322
}

and

ANDERSON D. SMITH

Georgia Institute of Technology, Atlanta, Georgia 30332

\begin{abstract}
Multitrial free recall was compared between groups engaging in semantic orienting tasks on each study trial and a standard group. When study and recall phases alternated, the standard group showed a steeper growth rate over trials, although the usual equivalence among groups after a single trial was replicated. This outcome was predicted on the assumption that the growth of recall with repetition depends on organizational encoding and that semantic orienting tasks emphasize the coding of individual items. When there was only a single test trial following three study trials, no difference was found, implicating an important role for retrieval processes in potentiating organizational encoding.
\end{abstract}

It is a common finding in the recent literature that more words will be recalled from a list following semantic processing of each word than following nonsemantic processing. While much has been made of this finding, we are equally impressed by the frequent finding that as many words are recalled after standard intentional learning instructions as after semantic orienting tasks (see Hyde, 1973; Hyde \& Jenkins, 1969, 1973; Johnston \& Jenkins, 1971; Walsh \& Jenkins, 1973). That is, just telling people to try to learn the words on the list produced recall in these studies equivalent to that following semantic processing of each word in one way or another, for example, rating each word for pleasantness. This paper concerns the interpretation of this equivalence. One possibility is that, when told to remember a list of words, young adults engage in semantic processing and essentially do the same kind of encoding as subjects forced to engage in a semantic orienting task. Aside from obvious difficulties with this position (i.e., it seems unlikely that subjects spontaneously judge words for pleasantness or size), there is an empirical difficulty. If memory is tested with a recognition test, a different pattern emerges: Recognition memory, unlike recall, is facilitated following semantic orienting tasks vis a vis standard instructions (see Eagle \& Mulliken, 1974; Elias \& Perfetti, 1973; Griffith, 1975; Groninger, Bell, Cymer, \& Weiss, 1972). It is hard to argue that the encoding operations leading to this difference in recognition memory are functionally equivalent. How do they differ?

We will argue here that, with standard instructions,

This research was supported in part by National Institutes of Health Research Grant AG-00445-06 from the Institute on Aging to the second author. Susan E. Mason is acknowledged for assistance in this research. Requests for reprints should be addressed to Eugene Winograd, Department of Psychology, Emory University, Atlanta, Georgia 30322. subjects implicitly expect a recall test and prepare for it by organizing; that is, they look for associations between items and attempt to structure the list (Mandler, 1967; Tulving, 1962). On the other hand, semantic orienting tasks lead to elaborative coding of individual items with relatively less information stored about interitem relationships. On the assumption that recognition is enhanced more by elaborative encoding than is recall, superior recognition (relative to a standard condition) following semantic orienting tasks is to be expected. The problem with this line of reasoning is apparent when recall is considered, however. If standard instructions lead to a greater degree of organizational encoding than semantic orienting tasks, why do they lead to equivalent amounts of recall?

We present here data from an experiment showing the hypothesized superiority in free recall following standard instructions; the difference between our work and previous studies is that we employed a multitrial, rather than a single-trial, procedure. It seems reasonable to argue that the single-trial recall experiment lacks sensitivity to organizational encoding, particularly when unrelated word lists are used. In many studies, recall following a single study trial is not very high and a substantial proportion of the observed recall reflects an invariant memory span or primary memory component, if Murdock (1961) is correct. In addition, organizational coding with unrelated word lists may take more than a single trial to get under way, perhaps because potentiation from retrieval processes taking place during output is necessary to induce relational encoding. For these reasons, we compared multitrial recall between subjects who engaged in semantic orienting tasks and subjects given standard intentional learning instructions. We predicted that the standard group would demonstrate more rapid growth in recall across trials.

In another group, recall was compared between se- 
mantic processing and standard groups following three successive study trials without interpolated recall tests in order to explore the possible role of test trials with these encoding treatments.

\section{METHOD}

\section{Subjects}

Two different intact class sections of the same introductory psychology course at the Georgia Institute of Technology participated in the experiments prior to a discussion of memory research. In one section, the subjects $(n=90)$ were presented a list of words three times followed by a single recall test, while the subjects in the other section $(n=69)$ recalled the list after each of the three presentations. One-third of the subjects in each section were given one of three different encoding instructions.

\section{Materials}

Thirty nouns with an average frequency of 10 were selected from the Thorndike and Lorge word count. Three random orders of the words were used for the three study trials.

\section{Procedure}

As indicated above, the subjects were tested in two different intact groups. One group heard the list three times followed by a single recall attempt, while the other group recalled the words after each list presentation. Within each group, subjects were assigned randomly to three different encoding conditions. One-third of the subjects were given standard intentional learning instructions. Of the remaining subjects, half performed the same orienting task on all three study trials (Same) and half performed different orienting tasks on each trial (Different). The orienting tasks were rating the words for pleasantness, rating the vividness of the image elicited by the word, and rating the number of associations estimated to each word. For each task subjects marked their ratings on a 5-point scale. The order of the three tasks in the Different condition as well as the type of task used in the Same condition were counterbalanced across subjects. The subjects were told what task to perform and how to do it by means of an instruction sheet prior to the presentation of the list on each trial. Since subjects engaged in an orienting task had to write something in their answer booklet for each word, subjects in the Standard condition were asked to check off the number of each word as it was presented. All subjects were expecting a later memory test. The number of the word in the sequence and the word itself were read aloud together by the experimenter at a 5-sec rate, with a timer used to pace the experimenter. For all recall tests, the instruction sheet asked that as many of the words as possible be written down in the booklet. A separate page was used for each recall test and $3 \mathrm{~min}$ were allowed for each test.

\section{RESULTS}

The results are summarized in Figure 1. A separate analysis with trials as a repeated measure was performed on the multitrial free recall data. The major result of this analysis was a significant interaction between orienting instructions and trials $[\mathrm{F}(4,132)=8.24$, $p<.01]$. The main effect of trials was significant $[F(2,132)=156.42]$, but no main effect of instructional condition was found. On Trial 1 , no differences were found between the three groups, in agreement with the single-trial recall studies cited earlier. However, recall scores increased in the different conditions across

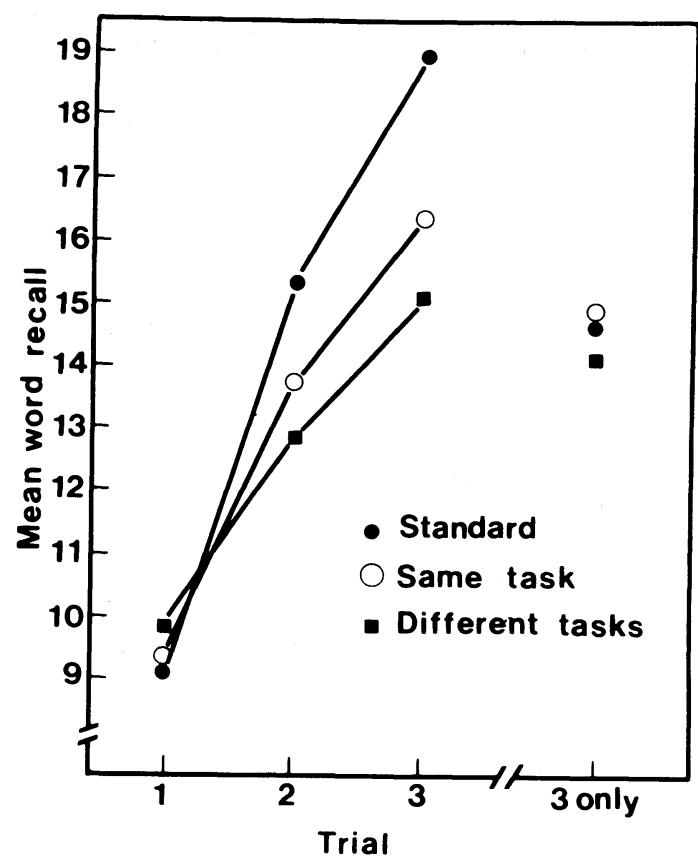

Figure 1. Mean number of words recalled over trials for subjects after different encoding tasks. The unconnected data points are from subjects recalling only after the third presentation of the list.

trials with different slopes, as the significant interaction indicates. By Trial 3, the Standard condition was performing best, followed by the Same condition and then the Different condition. Simple effects analyses showed that the groups differed on Trial $3[\mathrm{~F}(2,198)=4.64$, $p<.05$ ] but not on either Trial 1 or 2 , and Tukey ratios showed that each group differed from both of the other groups on Trial $3(\mathrm{p}<.05)$.

No significant effect of the Different task orders was found in the Different condition and the effect of the type of task used in the Same condition only approached significance $[F(2,24)=2.69, p<.10]$. The mean recall for the three different tasks in the Same condition were: pleasantness, 14.7; imagery, 13.5; and number of associations, 11.5. The curves for the three tasks over the three recall trials in the Same condition are parallel.

The amount of organization was examined using the pair-frequency measure of subjective organization advocated by Sternberg and Tulving (1977). The results of this organizational analysis are presented in Table 1. There was a significant increase in organization across trials $[\mathrm{F}(1,66)=17.12, \mathrm{p}<.01]$, and the difference between the conditions only approached significance $(p<.10)$. The predicted interaction between conditions and trials was found $[F(2,66)=3.28$, $\mathrm{p}<.05]$. As shown in the table, the Standard group showed a greater increase in organization across trials than either the Same or the Different group.

In contrast to the above findings, there was no 
Table 1

Mean Pair-Frequency Subjective Organization, Bidirectional O-E(ITR), in Recall

\begin{tabular}{lcc}
\hline & \multicolumn{2}{c}{ Trials } \\
\cline { 2 - 3 } Condition & $1-2$ & $2-3$ \\
\hline Same & .37 & .86 \\
Different & .08 & .31 \\
Standard & .12 & 1.18 \\
\hline
\end{tabular}

significant effect of encoding condition when a single recall test followed three presentations of the list. The recall means for these groups are shown as unconnected points in Figure 1.

\section{DISCUSSION}

The outcome of this experiment, when study and recall phases alternated in the conventional way, was as predicted: Recall grew at a faster rate with standard instructions than with semantic orienting tasks. This difference emerged even though the usual equivalence in single-trial recall among such groups was confirmed here after the first trial. We take this pattern as support for the hypothesis that orienting tasks which emphasize the encoding of individual words impede the growth of the kind of organization essential for the growth of recall with repetition. Unlike subjects given explicit orienting tasks, those in the standard group are free to go about their normal mnemonic business, which, to a considerable extent, consists of interrelating items with each other. This interpretation received strong additional support from the analysis of the subjective organization scores.

This interpretation of the greater growth of recall under standard intentional learning instructions should not be taken to mean, however, that organization plays no role in the growth of recall for the subjects engaged in semantic orienting tasks. In fact, the subjective organization scores increased across trials in all three conditions, as did recall. Postman and Kruesi (1977) recently made a case against the assumption that subjects performing orienting tasks are wholly occupied in processing the current item. Our point is that there is less organizational encoding (or displaced rehearsal, in Postman and Kruesi's terms) when subjects are engaged in an orienting task than when they are not given an explicit task to perform and, furthermore, that the single-trial recall experiment is not sensitive to this difference in the degree of organizational encoding.

The finding of greater recall on Trial 3 for the Same condition over the Different raises some interesting issues. In related work Klein and Saltz (1976) had subjects judge words on two semantic dimensions and found that single-trial recall was higher when the dimensions were uncorrelated (e.g., pleasant-unpleasant and big-little) than when they were correlated (e.g., pleasantunpleasant and happy-sad). The present finding of greater recall when the judged dimensions were maximally correlated, as in the Same condition, does not appear to be consistent with the position taken by Klein and Saltz (1976) or with any theory which emphasizes the advantages of encoding variability.

The finding that the three encoding groups were equivalent in recall when tests wore not interspersed between successive study trials requires comment. One interpretation of this outcome is that organization at input requires potentiation from retrieval; that is, organization results from an interaction between encoding and retrieval. (See Cooper and Monk, 1976, for a review of testing effects.) If this analysis is valid, the Standard condition is at a particular disadvantage when there is no opportunity for testing to potentiate organization since, it has been emphasized here, its superiority derives from greater reliance upon organization. The present data are only suggestive in this regard and raise a question for further research to attempt to answer.

\section{REFERENCES}

Cooper, A. J. R., \& Monk, A. Learning for recall and learning for recognition. In J. Brown (Ed.), Recall and recognition. London: Wiley, 1976.

Eagle, M., \& Mulliken, S. The role of affective ratings in intentional and incidental learning. American Journal of Psychology, 1974, 87, 409-423.

Elias, C. S., \& Perfetti, C. A. Encoding task and recognition memory: The importance of semantic encoding. Journal of Experimental Psychology, 1973, 99, 151-156.

GRIFFITH, D. Comparison of control processes for recognition and recall. Journal of Experimental Psychology: Human Learning and Memory, 1975, 1, 223-228.

Groninger, L. D., Bell, B., Cymer, W., \& Weiss, B., JR. Storage aspects of nouns presented under imagery and acoustic coding instructions. Journal of Experimental Psychology, 1972, 95, 195-201.

HydE, T. S. Differential effects of effort and type of orienting task on recall and organization of highly associated words. Journal of Experimental Psychology, 1973, 97, 111-113.

Hyde, T. S., \& Jenkins, J. J. Differential effects of incidental tasks on the organization of recall of a list of highly associated words. Journal of Experimental Psychology, 1969, 82, 472-481.

Hyde, T. S., \& Jenkins, J. J. Recall for words as a function of semantic, graphic, and syntactic orienting tasks. Journal of Verbal Learning and Verbal Behavior, 1973, 12, 471-480.

Johnston, C. D., \& Jenkins, J. J. Two more incidental tasks that differentially affect associative clustering in recall. Journal of Experimental Psychology, 1971, 89, 92-95.

KleiN, K., \& SALTz, E. Specifying the mechanisms in a levels-of-processing approach to memory. Journal of Experimental Psychology: Human Learning and Memory, 1976, 2, 671-679.

MANDleR, G. Organization and memory. In K. W. Spence \& J. T. Spence (Eds.), The psychology of learning and motivation (Vol. 1). New York: Academic Press, 1967.

MURDock, B. B., JR. The retention of individual items. Journal of Experimental Psychology, 1961, 62, 618-625.

Postman, L., \& Kruesi, E. The influence of orienting tasks on the encoding and recall of words. Journal of Verbal Learning and Verbal Behavior, 1977, 16, 353-369.

Sternberg, R. J., \& Tulving, E. The measurement of subjective organization in free recall. Psychological Bulletin, 1977, 84, 539-556.

Tulving, E. Subjective organization in free recall of "unrelated” words. Psychological Review, 1962, 69, 344-354.

WALSH, D. A., \& JENKINS, J. J. Effects of orienting tasks on free recall in incidental learning: "Difficulty," "Effort," and "Process" explanations. Journal of Verbal Learning and Verbal Behavior, 1973, 12, 481-488.

(Received for publication November 15, 1977.) 